\title{
An improved scan mode in an electrostatic force microscope for surface profile measurement of micro-optics
}

\author{
Zhigang JIA*, Keiichiro HOSOBUCHI*, So ITO*, Yuki SHIMIZU* and Wei GAO* \\ * Department of Nanomechanics, Tohoku University \\ 6-6-01, Aramaki Aza Aoba, Aoba-ku, Sendai 980-8579, Japan \\ E-mail: so.ito@nano.mech.tohoku.ac.jp
}

Received 28 February 2014

\begin{abstract}
A new scan mode referred to as the feed-forward controlled unidirectional scan mode is employed to improve the accuracy of surface profile measurement of micro-optics in a scanning electrostatic force microscope (SEFM) instead of the conventional feedback controlled bidirectional scan mode. Two kinds of error sources, the positioning deviation of the X-stage and the frequency shift detection delay due to the lock time of the PLL circuit, are analyzed theoretically. It is verified that large profile measurement error can be introduced by the error sources when steep structures are included in the surface profile. It is also demonstrated that the feed-forward controlled unidirectional scan mode can remove the influence of the positioning deviation error component. It can also reduce the influence of the frequency shift detection delay error component by shortening the lock time of the PLL circuit and increasing the bandwidth of the PLL circuit through a feed-forward control strategy of the scan traces with the PI controller of the Z-scanner turned off. Experiments of surface profile measurement are carried out on two diffraction grating samples with different grating structures. The feasibility of the new scan mode is confirmed by the experimental results.
\end{abstract}

Key words : Accuracy, EFM, Scan, Surface, Profile, Measurement, Micro-optics

\section{Introduction}

Diffractive and refractive micro-optics, which have periodical large amplitude microstructures with steep local slopes on their surfaces, are key components that have been widely employed in precision nanometrology and manufacturing systems (Bruzzone, et al., 2008). Quantitative and non-destructive dimensional metrology of the microstructures, for example, the pitch and the amplitude as well as the three-dimensional (3D) surface profile is indispensable for quality control in the process of manufacturing the micro-optics (Xu, et al., 2013). Since a lateral resolution of 10 to $100 \mathrm{~nm}$ and a vertical resolution of 1 to $10 \mathrm{~nm}$ are demanded for such a measurement, scanning probe microscope (SPM) is one of the best measuring instruments to fulfill the requirement. The representative types of SPM are noncontact atomic force microscopes (NC-AFMs) and scanning tunneling microscopes (STMs), which can make noncontact nanometric 3D surface profile measurement (Baykara, et al., 2010). However, the tiny tip-to-sample distances, typically smaller than $10 \mathrm{~nm}$, on account of the weak tip-sample interactions of Van der Waals force or tunneling current make it difficult for the NC-AFMs and STMs to measure the micro-optics with steep surface slopes because of the high possibility of collision between the tip and the sample (Danzebrink, et al., 2006). It is thus expected to have a SPM with a large enough tip-to-sample distance for surface profile measurement of the micro-optics. On the other hand, the long-range electrostatic force is utilized in the electrostatic force microscope (EFM), which can be detected even when the tip-to-sample distance is larger than $100 \mathrm{~nm}$ for evaluating the electric properties of a sample with a flat surface or a known profile (Girard. 2001). However, the conventional EFMs have not been employed for surface profile measurement because the electric property information and surface profile information of the sample surface are mixed in the electrostatic force signals.

Recently, the authors have developed a new scanning electrostatic force microscope (SEFM) for noncontact surface profile measurement (Gao, et al., 2012). A dual height method has been proposed to separate the surface profile 
information from the electric property information in the electrostatic force signal. This method is realized by a bidirectional scan mode. In this scan mode, an X-directional cross-section of the sample surface, which is referred to as the X-line, is first scanned by the probe along the positive X-direction. At the end of the first forward scan trace, which is referred to as the lower trace, the probe tip is lifted up to a higher position along the Z-direction by the Z-scanner of the probe. Then the same X-line is scanned again by the probe along the negative X-direction. This backward scan trace is referred to as the upper trace. During each of the scans, the corresponding frequency shift from the resonance frequency of the oscillating probe, which is proportional to the gradient of the electrostatic force, is kept to a certain value through feedback control of the Z-directional positions of the probe by the Z-scanner, which is referred to as the constant frequency-shift scanning. The surface profile of the sample can then be obtained from the Z-displacements of the Z-scanner in the two scans. For the constant frequency-shift scanning, a phase-locked loop (PLL) circuit is employed to detect the frequency shift signal. The frequency shift signal is sent to a PI controller of the Z-scanner for the feedback control of the Z-directional probe position. However, an inappropriate setting of the gains of the PID controller of the PLL circuit will generate a delay in the frequency shift detection, especially for a high scanning speed (Gildemeister, et al., 2007). The delayed frequency shift signal induces errors in the measured surface profile by the dual height method. The position deviations of the X-directional scanning stage between the upper and lower traces also deteriorate the accuracy of the measured surface profile.

In order to improve the accuracy of the measured surface profile, a new scan mode, which is referred to as the feed-forward controlled unidirectional scan mode, is presented in this paper. In this scan mode, the same X-line of the sample is scanned twice with different tip-to-sample distances but the same scanning direction so that the influence of the positioning deviations of the X-stage on the surface profile measurement based on the dual height method can be avoided. To remove the influence of the frequency shift detection delay caused by the lock time of the PLL circuit, a feed-forward control strategy is employed to combine with the unidirectional scan mode, by which the bandwidth of the PLL circuit can be increased and thus the lock time can be shortened. Experiments are carried out to demonstrate the effectiveness of the feed-forward controlled unidirectional scan mode.

\section{Principle of the feed-forward controlled unidirectional scan mode}

Figure 1 shows a schematic of the SEFM system. Two identical probes are glued on a tuning fork quartz crystal resonator (TF-QCR) and vibrating together at their resonance frequency $f_{0}$. One of the probes works as the measuring probe and the other as the counterweight. The oscillating performance of the resonator can be improved by adding the counterweight probe (Ito, et al., 2013). The oscillating unit (the probe and the TF-QCR) is driven by a long-stroke ultra-high precision Z-scanner of piezoelectric type in which a linear encoder is employed for compensation of the

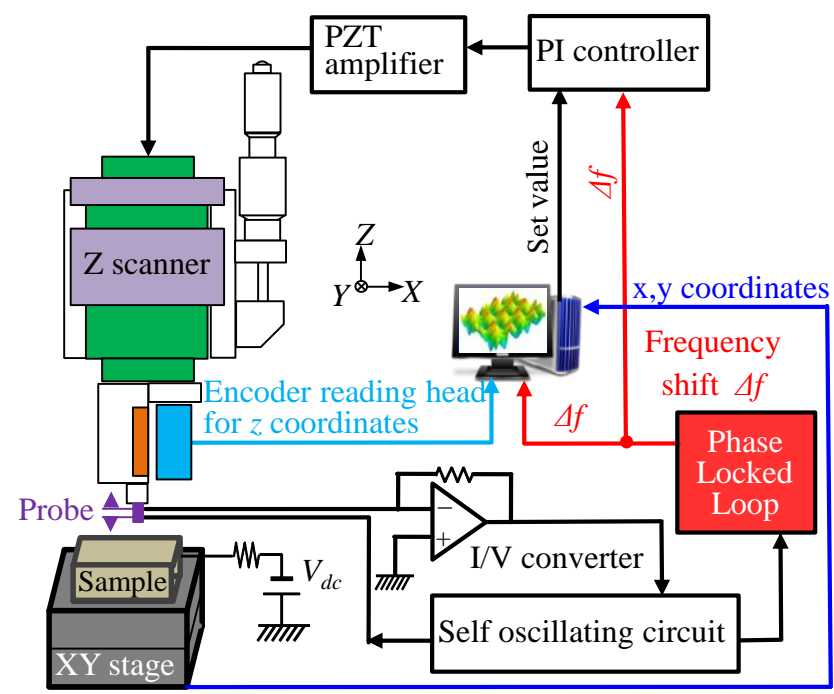

Fig. 1 Schematic of the scanning electrostatic force microscope (SEFM) system 


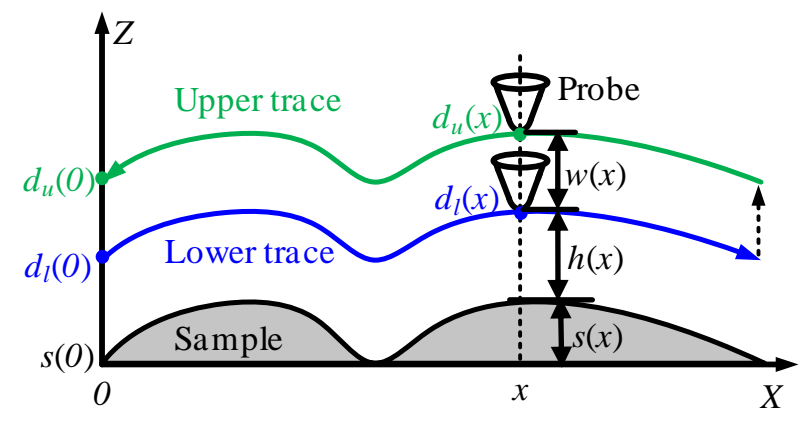

(a) Bidirectional scan mode

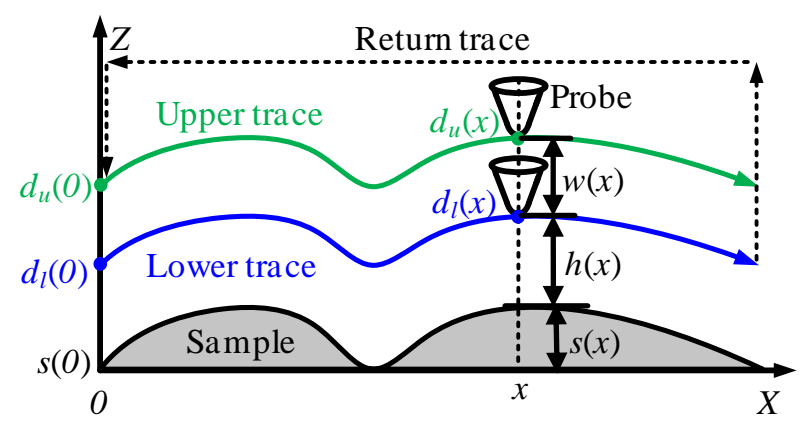

(b) Unidirectional scan mode

Fig. 2 Bidirectional scan mode and unidirectional scan mode

Z-directional displacement errors of the scanner. The Z-scanner has an effective measurement range of $50 \mu \mathrm{m}$ with a non-linearity error less than $10 \mathrm{~nm}$ as well as a least significant bit resolution of $0.5 \mathrm{~nm}$ (Goto, et al., 2011). The sample is fixed on a PZT driven XY-stage. The bias voltage $V_{d c}$ is applied between the probe and the sample surface to produce an electrostatic force between them. The resonance frequency of the oscillation unit will be changed by the electrostatic force when the probe approaches close to the sample surface. In consequence, the frequency of the oscillating unit is shifted and the shift amount $\Delta f$ can be expressed by:

$$
\Delta f(x) \approx \frac{f_{0}}{2 k} F^{\prime}(x)=-\frac{\pi \varepsilon_{0} \varepsilon_{r} R f_{0}}{2 k}\left(V_{d c}+V_{c p d}(x)\right)^{2} \frac{1}{h^{2}(x)}
$$

where $\varepsilon_{0}$ and $\varepsilon_{\mathrm{r}}$ are the dielectric constant of vacuum and the relative static permittivity, respectively. $k$ is the spring constant of the oscillating unit. $R$ is the apex radius of the probe tip. $F^{\prime}(x)$ represents the electrostatic force gradient. $V_{d c}$ is the bias voltage and $V_{c p d}$ expresses the contact potential difference (CPD) between the probe tip and the sample surface. $h(x)$ is regarded as the tip-to-sample distance.

The frequency shift signal is detected by utilizing a commercial phase-locked loop (PLL) circuit (Nanonis OC4 oscillation controller). The frequency shift signal is recorded by a personal computer (PC) and fed into the PI controller of the Z-scanner to make sure the probe scans the sample surface with a constant frequency shift, which is similar with the frequency modulation (FM) AFMs.

From Eq. (1), it can be seen that the magnitude of the frequency shift is not only related to the tip-to-sample distance $h$ but also affected by $V_{c p d}$ in Eq. (1), which is determined by the electric properties of the sample surface. To separate $h$ from $V_{c p d}$, as shown in Fig. 2(a), another scan of the same X-line, which is started at the end point of the lower trace, is carried out at a higher $\mathrm{Z}$ position with the reversed scan direction to the lower trace. This scan mode for the dual height method is referred as the bidirectional scan mode.

The frequency shift at the upper trace can be evaluated as:

$$
\Delta f_{u}(x) \approx \frac{f_{0}}{2 k} F_{u}^{\prime}(x)=-\frac{\pi \varepsilon_{0} \varepsilon_{r} R f_{0}}{2 k}\left(V_{d c}+V_{c p d}(x)\right)^{2} \frac{1}{(h(x)+w(x))^{2}}
$$

where $w(x)=d_{u}(x)-d_{l}(x)$ is the gap between the upper trace and the lower trace. $d_{u}(x)$ and $d_{l}(x)$ are the outputs of the linear encoder of the Z-scanner which express the relative height changes of the probe tip in the Z-direction during the scans. The electric property is supposed not to change at the same $x$ position of the two traces if the gap $w(x)$ is very small. As a result, the correct tip-to-sample distance without the influence of the electric property distribution can be calculated as:

$$
h(x)=w(x) \frac{\sqrt{\Delta f_{u}(x) / \Delta f_{l}(x)}}{1-\sqrt{\Delta f_{u}(x) / \Delta f_{l}(x)}}
$$




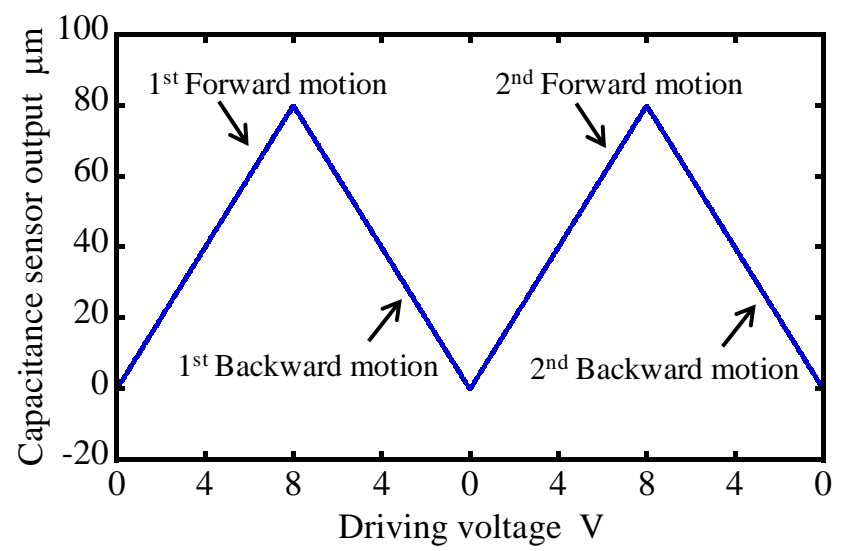

Fig. $3 \mathrm{X}$-stage positioning accuracy between forward motion and backward motion

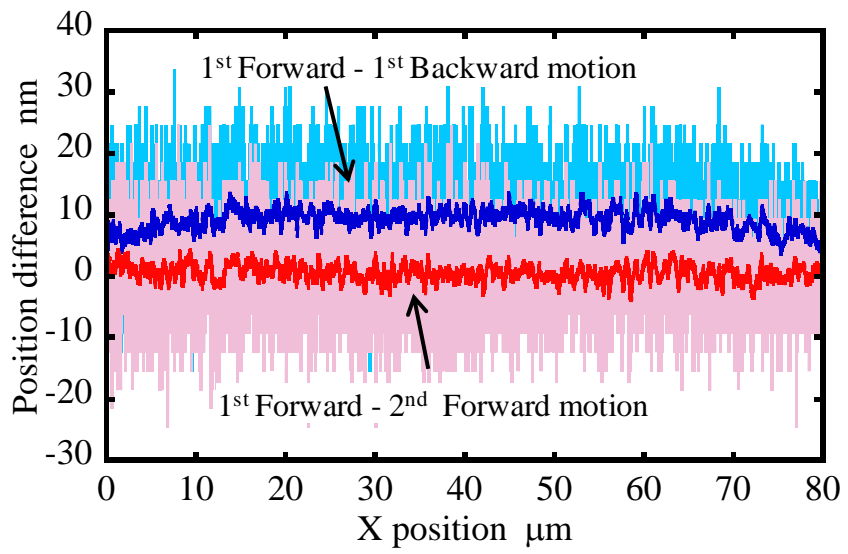

Fig. 4 Positioning difference between forward-forward scan and forward-backward scan

The surface profile can be written as:

$$
s(x)=d_{l}(x)-h(x)
$$

According to the principle of the dual height method, the accuracy of the evaluated surface profile is significantly influenced by the position coincidence at the same $x$ position between the lower trace and the upper trace. Furthermore, the sampling of $\Delta f(x), d_{l}(x)$ and $d_{u}(x)$ at the corresponding $x$ position without any signal detection delays at each scanning trace is also important for the accurate calculation of the surface profile.

Although the PZT XY-stage utilizes capacitance displacement sensors to realize closed-loop precision positioning in XY-directions, the positioning deviation exists between the forward trace and the backward trace along the $\mathrm{X}$-direction when the bidirectional scan mode for the dual height method is carried out. Verification experiment of the $\mathrm{X}$-stage positioning accuracy is carried out. As shown in Fig. 3, the X-stage was driven forward and backward twice with a range of $80 \mu \mathrm{m}$, and the output of the capacitance sensor of the X-stage was recorded and shown in Fig. 3. Next, the positioning deviation between the first forward and first backward motion was calculated and displayed in Fig. 4. The positioning deviation between two forward motions is also shown in Fig. 4, in which the bright blue line and the pink line show the raw data and the dark blue line and the red line show the corresponding 30 points moving-averaged value. It can be seen that the positioning deviation between the forward scans almost equals zero. On the other hand, the positioning deviation between the forward and the backward scans is approximate $10 \mathrm{~nm}$, which will introduces errors in the calculated profile when the bidirectional scan mode for the dual height method is carried out.

To solve the problem, the unidirectional scan mode, as shown in Fig. 2(b), is employed to reduce the errors of surface profile measurement caused by the positioning deviation of the X-stage in the bidirectional scan mode. Differing from the bidirectional scan mode, the upper trace of the unidirectional scan mode has the same starting point 


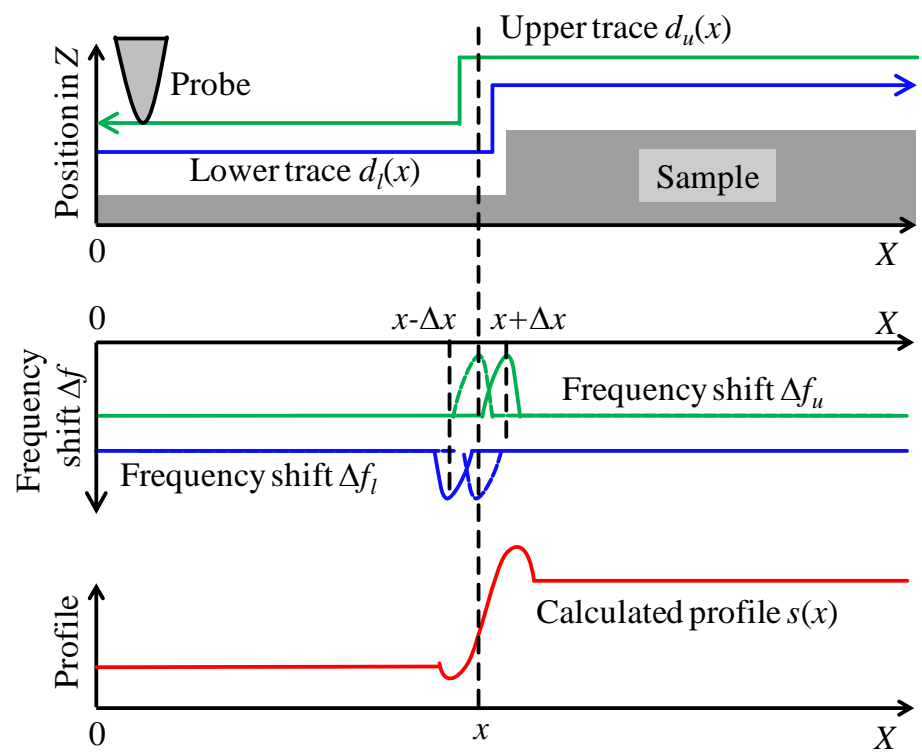

Fig. 5 Surface profile errors caused by frequency shift detection delay

of $x=0$ and the same scan direction (forward direction) as the lower trace. However, at the end position of the lower trace, the probe is lifted upward to a safe position which is far away from the sample surface. The probe is then driven by the $\mathrm{X}$-stage along the return trace to the starting point of $x=0$ with a rapid speed. After that, the probe is moved to the starting point of $d_{u}(0)$ by the Z-scanner for the upper trace scan.

As described previously, another error source which influences the profile measurement accuracy comes from the frequency shift detection delay associated with the scanning motion. This is caused by an inappropriate setting of the gains of the PI controller in the PLL circuit, which results in a slow response and a long lock time when the frequency shift signal is generated. As shown in Fig. 5, at the lower trace, when the probe scans at the $x$ position, the frequency shift signal at $x-\Delta x$ will be sampled if the frequency shift detection delay exists. On the other hand, at the upper trace of the bidirectional scan mode, the frequency shift signal at $x+\Delta x$ will be sampled when the probe scans at the $x$ position. Since the Z-scanner is feedback controlled with the frequency shift signal, the detection delay of the frequency shift signal also introduces delays in sampling $d_{l}(x)$ and $d_{u}(x)$. As a consequence, the surface profile which is calculated by the delayed sampling data can be expressed as:

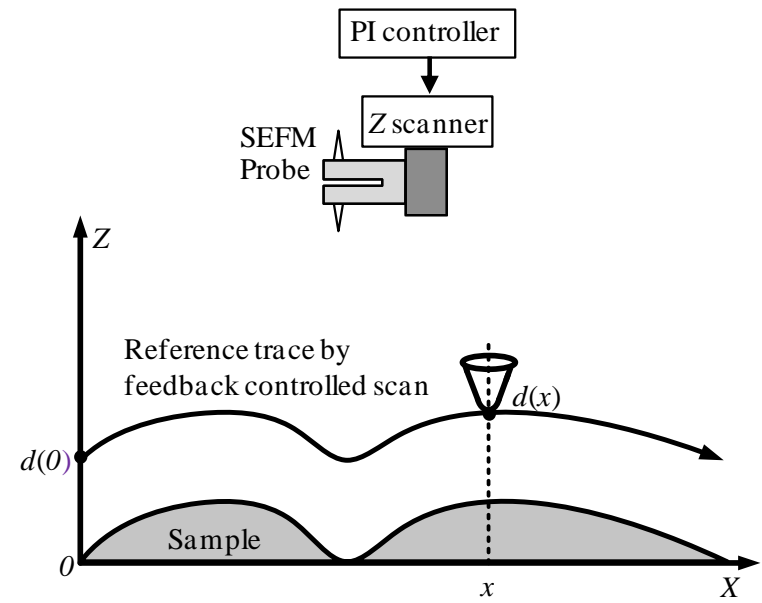

(a) Step 1

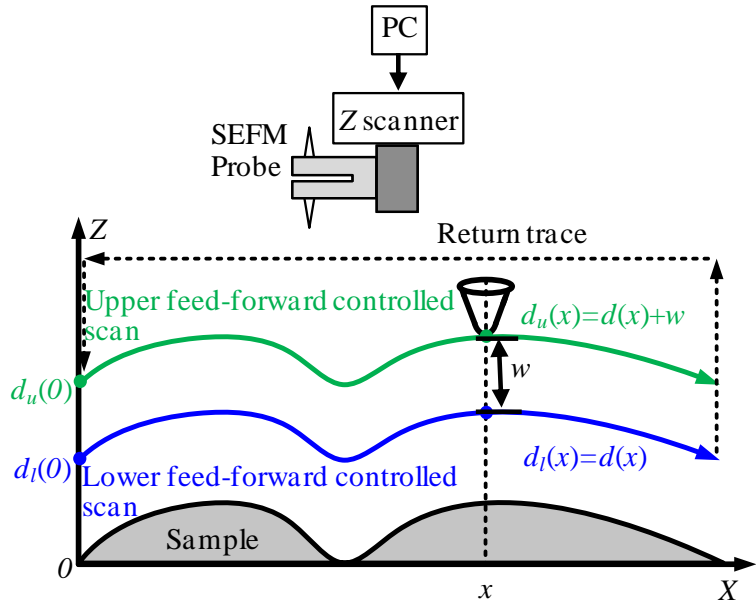

(b) Step2

Fig. 6 The process of the feed-forward controlled unidirectional scans 


$$
s_{\text {bidirectional }}(x)=d_{l}(x-\Delta x)-\left(d_{u}(x+\Delta x)-d_{l}(x-\Delta x)\right) \frac{\sqrt{\Delta f_{u}(x+\Delta x) / \Delta f_{l}(x-\Delta x)}}{1-\sqrt{\Delta f_{u}(x+\Delta x) / \Delta f_{l}(x-\Delta x)}}
$$

On the contrary, however, the surface profile by the unidirectional scan mode with consideration of the delayed sampling data can be written as:

$$
s_{\text {unidirectional }}(x)=d_{l}(x-\Delta x)-\left(d_{u}(x-\Delta x)-d_{l}(x-\Delta x)\right) \frac{\sqrt{\Delta f_{u}(x-\Delta x) / \Delta f_{l}(x-\Delta x)}}{1-\sqrt{\Delta f_{u}(x-\Delta x) / \Delta f_{l}(x-\Delta x)}}
$$

By comparing Eqs. (5) and (6), it can be seen that the calculation error of the surface profile by the unidirectional scan mode can be reduced for accuracy improvement of the measured surface profile although the frequency shift detection delay error exists in both the bidirectional scan mode and the unidirectional scan mode.

On the other hand, the lock time of the PLL circuit can be shortened by enlarging the bandwidth of the PLL circuit, however, a wide bandwidth will induce large noises in the locked frequency shift signal and reduce the $\mathrm{S} / \mathrm{N}$ ratio of the measurement. Since the frequency shift signal is fed into the PI controller of the Z-scanner, a large noise in the frequency shift signal will give rise to the oscillation of the probe tip position in the Z-direction and also increase the risk of tip-sample collision when scanning a surface with high aspect ratio topography. In the following, a feed-forward control strategy is employed to combine with the unidirectional scan mode for reducing the influence of the frequency shift detection delay by enlarging the bandwidth of the PLL circuit without the feedback control of the probe positions in the upper and lower traces for the dual height method. The process of the open loop scanning has two steps as shown

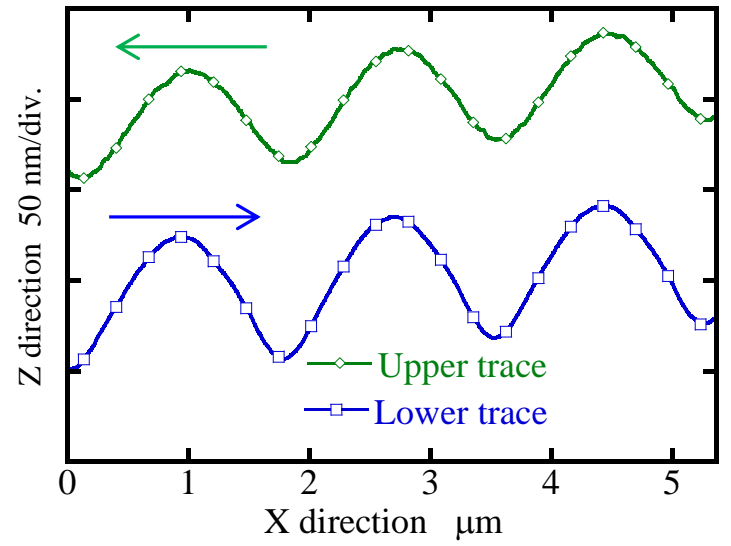

(a) Scanning traces

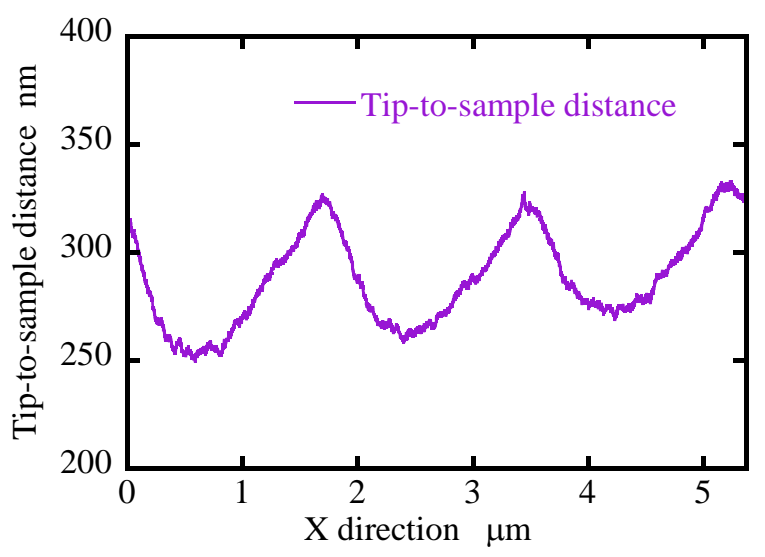

(c) Tip-to-sample distance

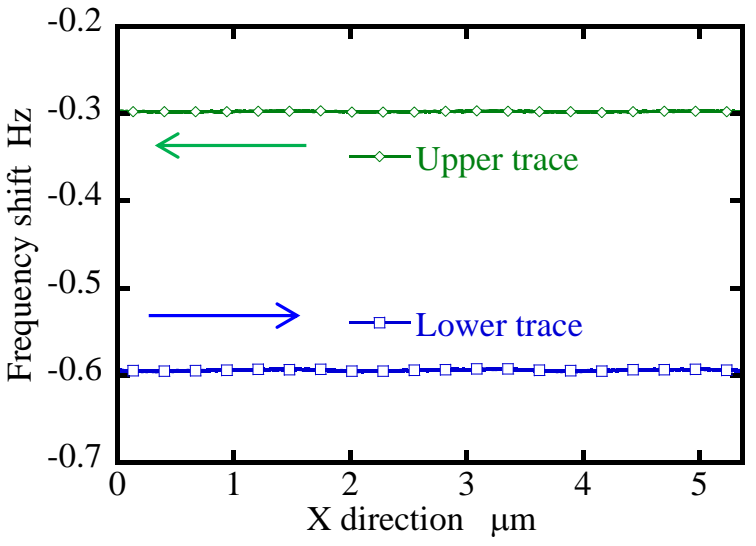

(b) Frequency shift signal

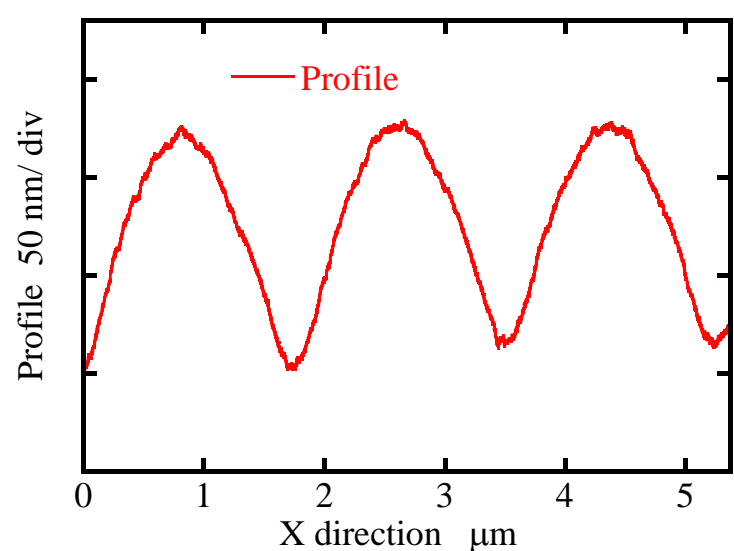

(d) Profile

Fig. 7 Measurement results of a blazed grating by the feedback controlled bidirectional scan mode 
in Fig. 6.

Step 1: Closed-loop controlled scan of an X-line with a small bandwidth setting of the PLL circuit. The probe trace of this scan is recorded by the PC and regarded as the reference trace for the following feed-forward scans.

Step 2: The PI controller of the Z-scanner is turned off and the position of the Z-scanner is directly controlled by the PC. The lower feed-forward controlled scan of the same X-line will be carried out at the starting point of $x=0$. The position of the Z-scanner at each $x$ position is adjusted by comparing the linear encoder output with the height data of the reference trace which has been recorded in Step 1. At the end of the lower feed-forward controlled scan, the probe is lifted up to a higher position and the $\mathrm{X}$-stage is moved back to the starting point of $x=0$. The upper feed-forward controlled scan will be carried out with the same starting point and the same scan direction as the lower feed-forward scan. The position of the Z-scanner at each $x$ position is also decided by the reference trace of Step 1 .

The bandwidth of the feed-forward controlled scan can be enlarged compared with the closed-loop controlled scan in Step 1 because the PI controller of the Z-scanner is turned off. As a result, the lock time of the PLL circuit can be shortened and the influence of the frequency shift detection delay can be reduced according to Eq. (6). Since the probe traces of the feed-forward controlled scan are based on the reference trace of the closed-loop controlled scan in Step 1, the gap $w(x)$ between the upper feed-forward controlled scan and the lower feed-forward controlled scan along the scanning direction is kept constant.

\section{Experiments}

Experiments of surface profile measurement were carried out on two gratings with different grating structures. The first sample was a gold coated blazed grating whose grating grooves had been diamond-cut by a ruling engine. The

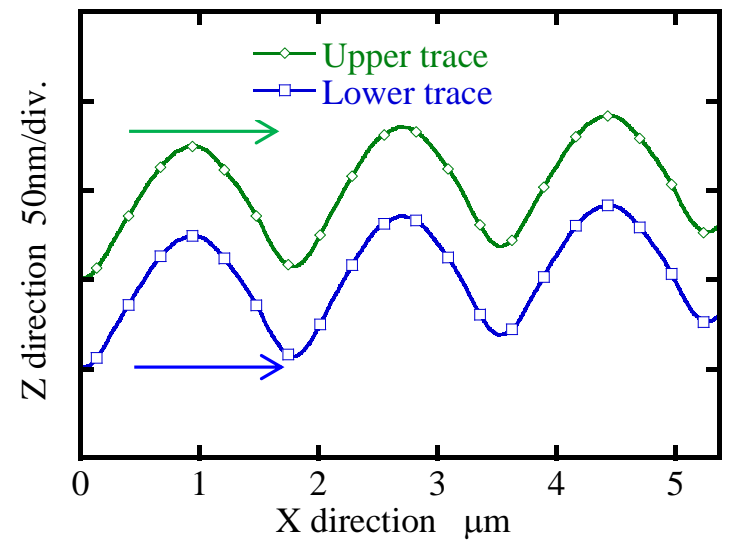

(a) Scanning traces

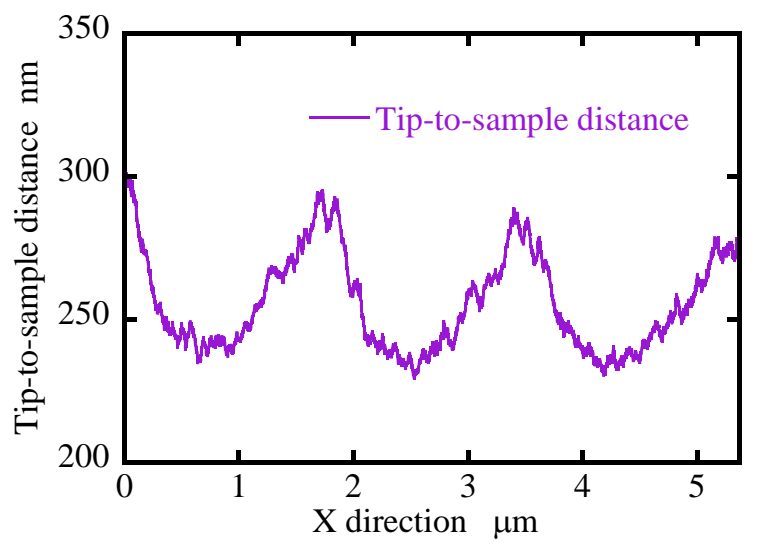

(c) Tip-to-sample distance

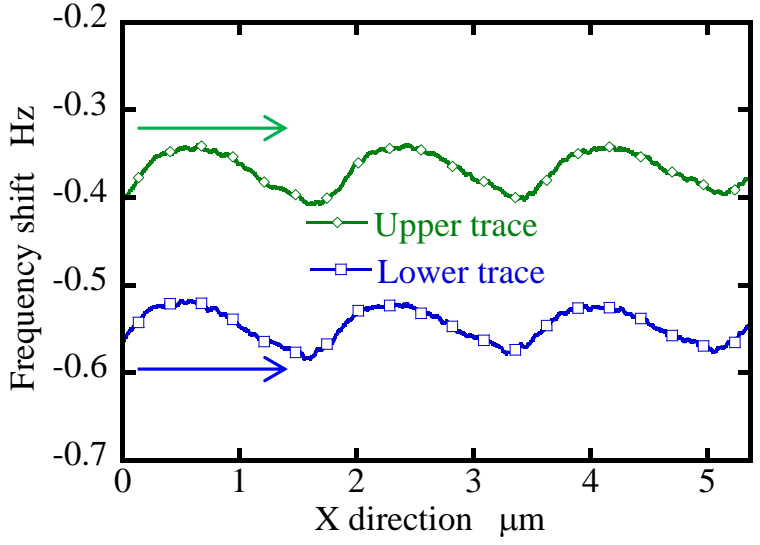

(b) Frequency shift signal

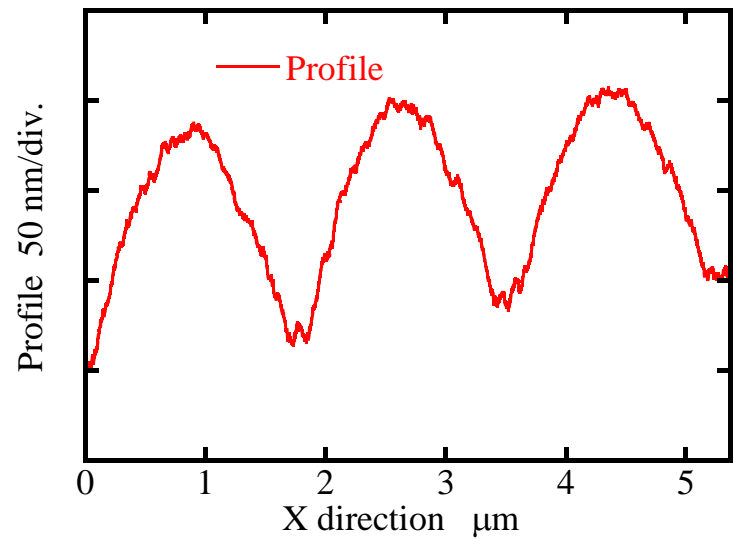

(d) Profile

Fig. 8 Measurement results of a blazed grating by the feed-forward controlled unidirectional scan mode 
nominal pitch of the grooves was approximately $1.67 \mu \mathrm{m}$. The groove grating structure had relatively gentle local slopes. The grating surface was firstly measured by the feedback controlled bidirectional scan mode. The bias voltage was set as $50 \mathrm{~V}$, and the $\mathrm{Q}$ value of the oscillation unit was 6100 , which provided a high sensitive detection of electrostatic force changes. The scanning pitch and range in the X-direction were $2 \mathrm{~nm}$ and $5.4 \mu \mathrm{m}$, respectively. The scanning speed was set to be $200 \mathrm{~nm} \cdot \mathrm{s}^{-1}$. The bandwidth of the PLL circuit was set to be $10 \mathrm{~Hz}$. The measurement result is shown in Fig. 7. Figure 7(a) shows the sampled linear encoder output which represents the relative probe height changes in the Z-direction. Since the scans were carried out in the feedback controlled mode with constant frequency shifts, the outputs of the linear encoder showed periodical changes in responding to the profile changes of the grating structures. Figure 7(b) shows the sampled frequency shift signals during the scans. It can be seen that the frequency shift signals were kept as constant values, which were referred to as the set points of the upper and the lower traces for the PI feedback controller of the Z-scanner. Figure 7(c) shows the calculated tip-to-sample distance based on the dual height method. It can be seen that the profile could be detected with a large tip-to-sample distance which was larger than $200 \mathrm{~nm}$. Figure 7(d) shows the calculate profile based on the dual height method. It had an average amplitude of $118 \mathrm{~nm}$.

The lower trace of the feedback controlled scan was regarded as the reference trace for the feed-forward controlled unidirectional scan. The scan was carried out with the same sampling numbers and scanning speed as the above feedback controlled bidirectional scan. However, the bandwidth of the PLL circuit was increased to $15 \mathrm{~Hz}$ to shorten the lock time of frequency shift signal. Figure 8(a) shows the feed-forward controlled scan traces. It can be seen that the lower trace and the upper trace had the same appearance as the reference trace. The trace height difference of feed-forward controlled scan was kept to be $50 \mathrm{~nm}$, which was set before the scan. Figure 8(b) shows the sampled

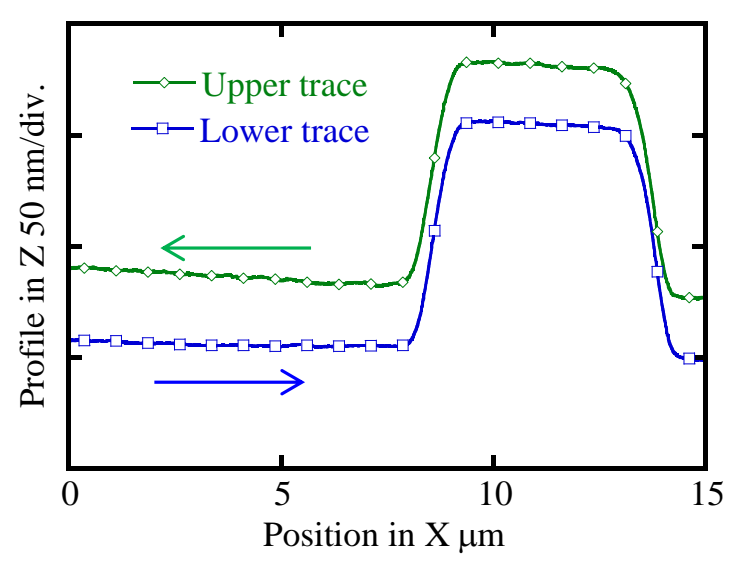

(a) Scanning traces

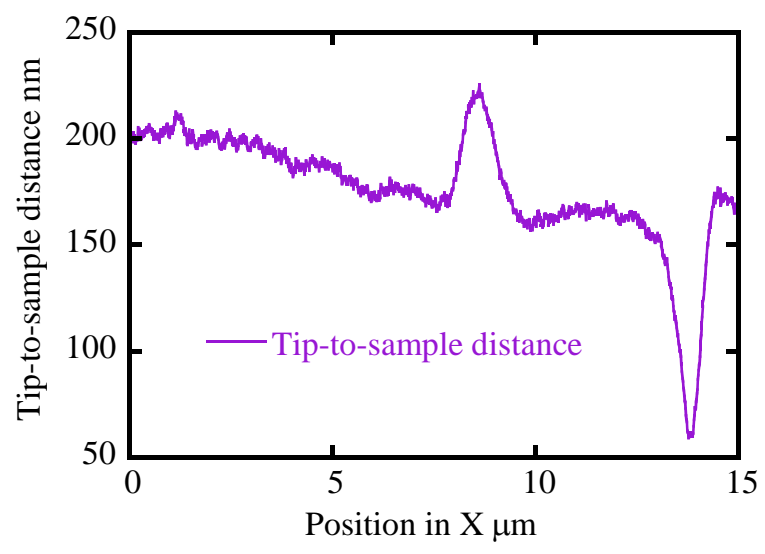

(c) Tip-to-sample distance

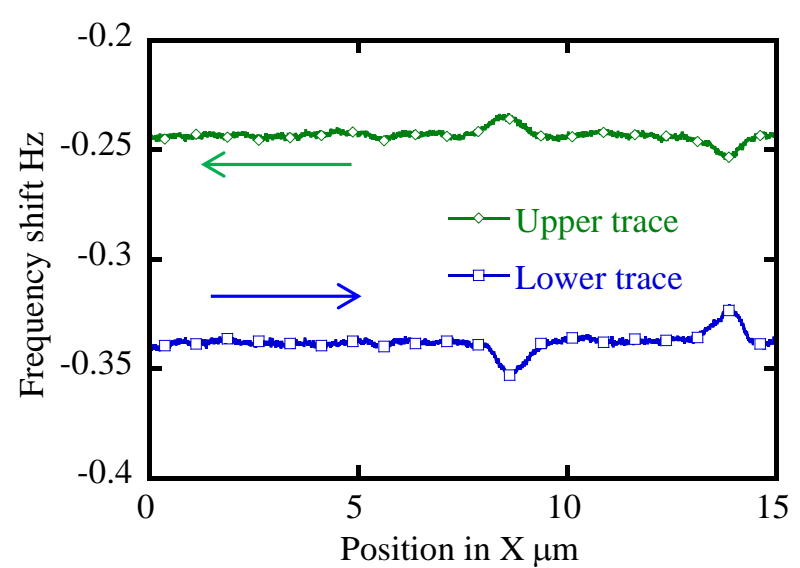

(b) Frequency shift signal

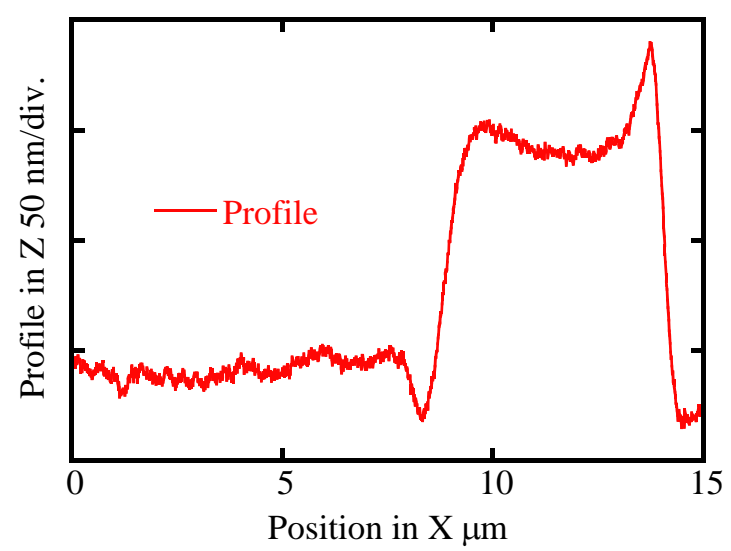

(d) Profile

Fig. 9 Measurement results of a rectangular grating by the feedback controlled bidirectional scan mode 
frequency shift signals during the scans. As the PI controller of the Z-scanner was turned off for the feed-forward control, the frequency shifts showed periodic changes with respect to those of the surface profile. Since the bandwidth of the PLL circuit was increased, larger noises could be observed in the sampled frequency shift signals. Figure 8(c) shows the calculated tip-to-sample distance, which was similar to that of the feedback controlled scan shown in Fig. 7(c). Figure 8(d) shows the measured profile by the dual height method. The average amplitude was $122 \mathrm{~nm}$. The measured surface profiles by the two scan modes were consistent to each other because the influence of the frequency shift detection delay was small for the sake of the small slope changes in the grating structures of the blazed grating.

The second sample was a rectangular grating with a step height grating structrue made by photolithography. The top and bottom areas of the step were made of $\mathrm{Au}$ and $\mathrm{Cr}$, respectively. The nominal height and width of the step were $100 \mathrm{~nm}$ and $6 \mu \mathrm{m}$, respectively. The local slopes at the rising and falling edges of the step were very steep, which could introduce large frequency shift detection delays during the scans. Figs. 9 and 10 show the measured profiles of the step height sample by the conventional feedback conrolled bidirectional scan mode and the new feed-forward controlled unidirectional scan mode, respectively. The scanning speed was set to be $200 \mathrm{~nm} \cdot \mathrm{s}^{-1}$ for the conventional feedback controlled bidirectional scan. The bandwidth of the PLL circuit for the feedback controlled bidirectional scan was set to be $10 \mathrm{~Hz}$. The lower trace of the bidirectional scan was taken as the reference trace for the feed-forward controlled unidirectional scan. The constant gap $w$ between the two traces of the feed-forward controlled scan was set to be $80 \mathrm{~nm}$. The scanning speed and the bandwidth of the PLL circuit of the feed-forward controlled unidirectional scan were increased to be $500 \mathrm{~nm} \cdot \mathrm{s}^{-1}$ and $20 \mathrm{~Hz}$, respectively. Since the enlarged bandwidth of the PLL circuit will reduce the S/N ratio of the measurement, the profile measured by the feed-forward controlled unidirectional scan mode was recalculated by 10 points moving-averaged value. It can be seen from Fig. 9 that there were large surface profile

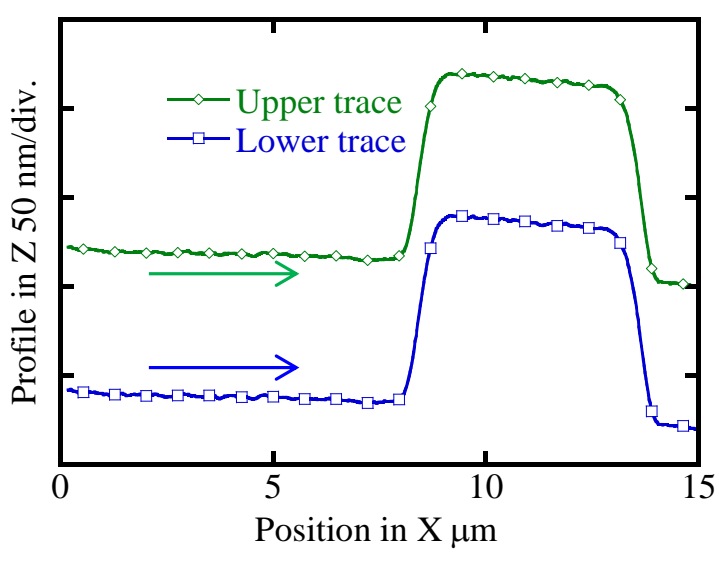

(a) Scanning traces

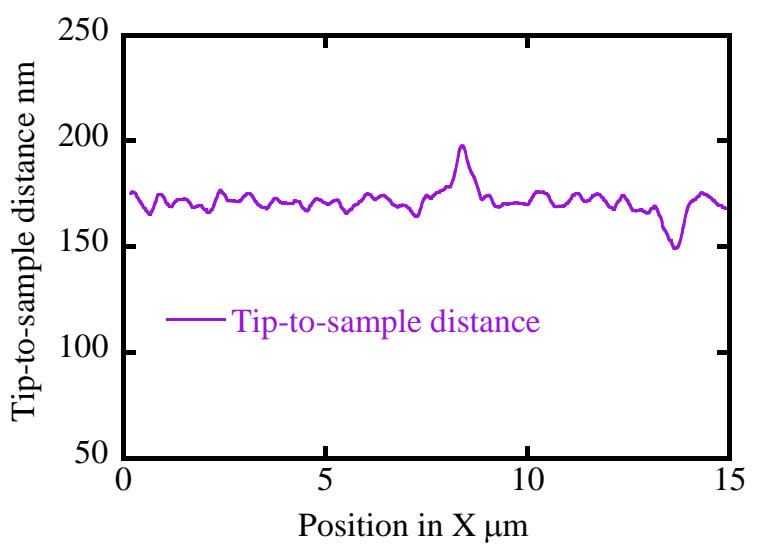

(c) Tip-to-sample distance

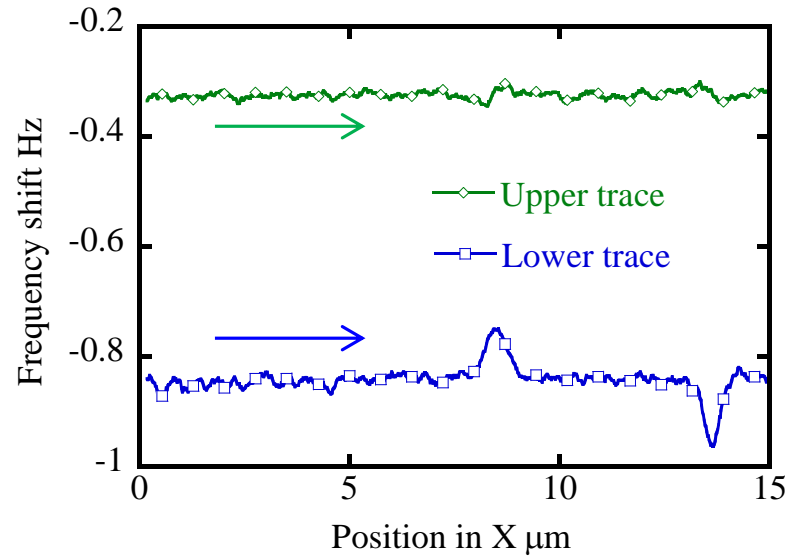

(b) Frequency shift signal

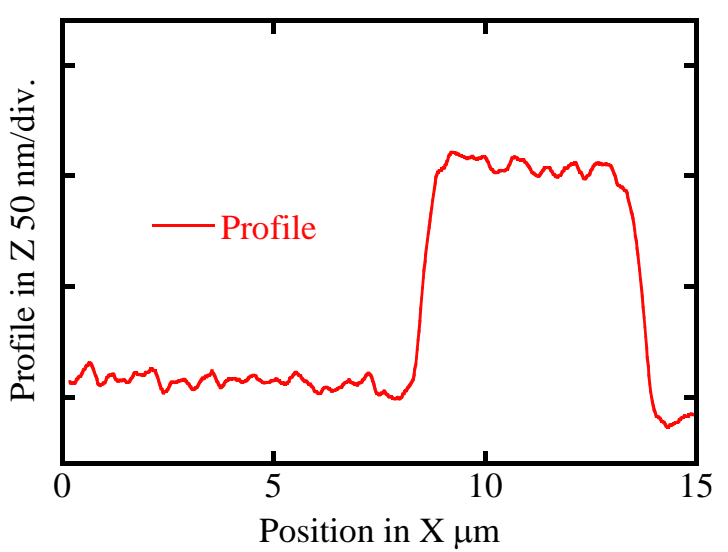

(d) Profile

Fig. 10 Measurement results of a rectangular grating by the feed-forward controlled unidirectional scan mode 
measurement errors with an amplitude of approximate $25 \mathrm{~nm}$ at the rising edge and $43 \mathrm{~nm}$ at the falling edge of the sample by the feedback controlled bidirectional scan mode. On the other hand the errors were removed by the feed-forward controlled unidirectional scan mode as shown in Fig. 10. The experiment results demonstrate the effectiveness of the feed-forward controlled unidirectional scan mode in surface profile measurement of micro-optics with steep slope changes in the dual height method-based SEFM.

\section{Conclusions}

A new feed-forward controlled unidirectional scan mode has been proposed to improve the profile measurement accuracy of a noncontact scanning electrostatic force microscope (SEFM) based on the dual height method for surface profile measurement of micro-optics. Compared with the conventional feedback controlled bidirectional scan mode, the new scan mode can remove the influence of the positioning deviation of the scanning stage between the two scan traces made at different height positions for calculation of the surface profile based on the dual height method. The influence of the frequency shift detection delay can also be improved by the new scan mode, in which the bandwidth of the PLL circuit can be set to a wider range for a shorter lock time to generate the frequency shift since the feed-back control of the probe height position for the constant frequency shift scan in the conventional feedback controlled bidirectional scan mode is no longer necessary.

In the experiments, the surface profiles of two diffraction gratings with different grating structures have been measured with the conventional feedback controlled scan mode and the new feed-forward controlled unidirectional scan mode. It has been demonstrated that the large measurement errors at the rising and falling edges of the rectangular grating structure caused by the conventional scan mode were significantly reduced by the new scan mode while the measurement results by the two scan modes were comparable for the blazed grating structure with small slope changes in the surface.

Further analysis of the characteristics of the new feed-forward controlled unidirectional scan mode as well as surface profile measurement experiments of micro-optics over large areas will be carried out as future work.

\section{Acknowledgement}

This research is supported by JSPS and Osawa Scientific Studies Grants Foundation.

\section{References}

Baykara, M. Z., Schwendemann, T. C., Altman, E. I. and Schwarz, U. D., Three-dimensional atomic force microscopy-taking surface image to the next level, Advanced Material, Vol.22, No.26-27 (2010), pp.2838-2853.

Bruzzone, A. A. G., Gosta, H. L., Lonardo, P. M. and Lucca, D. A., Advances in engineered surfaces for functional performance, Manufacturing Technology Annals of the CIRP, Vol.57 (2008), pp.750-769.

Danzebrink, H. U., Koenders, L., Wilkening, G., Yacoot, A. and Kunzmann, H., Advances in scanning force microscope for dimensional metrology, Manufacturing Technology Annals of the CIRP, Vol.55, No.2 (2006), pp.841-878.

Girard, P., Electrostatic force microscope: principles and some applications to semiconductors, Nanotechnology, Vol.12 (2001), pp.485-490.

Gao, W., Goto, S., Hosobuchi, K., Ito, S. and Shimizu, Y., A noncontact scanning electrostatic force microscope for surface profile measurement, Manufacturing Technology Annals of the CIRP, Vol.61 (2012), pp.471-474.

Gildemeister, A. E., Ihn, T., Barengo, C., Studerus, P. and Ensslin, K., Construction of a dilution refrigerator cooled scanning force microscope, Review of Scientific Instruments, Vol.78 (2007), 013704.

Goto, S., Hosobuchi, K. and Gao, W., An ultra-precision scanning tunneling microscope Z-scanner for surface profile measurement of large amplitude micro-structures, Measurement Science and Technology, Vol.22 (2011), 085101.

Ito, S., Jia, Z. G., Goto, S., Hosobuchi, K., Shimizu, Y., He, G. F. and Gao, W., An electrostatic force probe for surface profile measurement in noncontact condition, International Journal of Automation Technology, Vol.7, No.6 (2013), pp.714-719.

Xu, B., Jia, Z. G., Li, X. H., Chen, Y. L., Shimizu, Y., Ito, S. and Gao, W., Surface form metrology of micro-optics, Proceedings of SPIE, Vol.8769 (2013), 876902. 